\title{
Diabetic Macular Edema-Like Ocular Lesions in Male Spontaneously Diabetic Torii Fatty Rats
}

\author{
Y. MOTOHASHI ${ }^{1,2}$, Y. KEMMOCHI ${ }^{2,3}$, T. MAEKAWA $^{1}$, H. TADAKI $^{1}$, T. SASASE $^{1}$, \\ Y. TANAKA ${ }^{4}$, A. KAKEHASHI ${ }^{4}$, T. YAMADA ${ }^{2}$, T. OHTA $^{1}$ \\ ${ }^{1}$ Biological/Pharmacological Research Laboratories, Central Pharmaceutical Research Institute, \\ Japan Tobacco Inc., Osaka, Japan, ${ }^{2}$ Graduate School of Science and Technology, Niigata \\ University, Niigata, Japan, ${ }^{3}$ Toxicology Research Laboratories, Central Pharmaceutical Research \\ Institute, Japan Tobacco Inc., Hadano, Japan, ${ }^{4}$ Department of Ophthalmology, Saitama Medical \\ Center, Jichi Medical University, Saitama, Japan
}

Received July 7, 2017

Accepted December 15, 2017

On-line March 12, 2018

\begin{abstract}
Summary
Diabetic macular edema (DME) is a major factor contributing to visual disabilities in diabetic patients, and the number of patients is increasing. Animal models play a key role in the development of novel therapies. In this study, pathophysiological analyses of ocular lesions in Spontaneously Diabetic Torii (SDT) fatty rats were performed. First, vascular endothelial growth factor (VEGF) concentrations in vitreous humor, retinal vascular permeability and retinal thickness were measured in SDT fatty rats (Experiment 1). Furthermore, the pharmacological effects of two anti-diabetic drugs, phlorizin and pioglitazone, on retinal lesions were evaluated (Experiment 2). As results, the SDT fatty rats exhibited VEGF increase in vitreous humor at 8 and 16 weeks of age, and both retinal vascular hyperpermeability and retinal thickening at 16 weeks of age. In particular, the layers between the retinal internal limiting membrane and the outer nuclear layer were thickened. Phlorizin treatment from 4 to 16 weeks of age improved hyperglycemia and normalized retinal thickness; however, the effect of pioglitazone on retinal thickness was not strong despite the normalization of hyperglycemia. These data demonstrate that the male SDT fatty rat is a useful model for developing new therapeutic approaches in DME.
\end{abstract}

\section{Key words}

SDT fatty rat • VEGF • Diabetic retinopathy • Diabetic macular edema • Retinal thickening

\section{Corresponding author}

Y. Motohashi, Biological/Pharmacological Research Laboratories,
Central Pharmaceutical Research Institute, Japan Tobacco Inc., 1-1 Murasaki-cho, Takatsuki, Osaka 569-1125, Japan. E-mail: yu.motohashi@jt.com

\section{Introduction}

Diabetic macular edema (DME) is a frequent finding associated with diabetic retinopathy (DR) and is a major factor contributing to visual disabilities in patients with diabetes mellitus (Cheung et al. 2010). The current worldwide estimates are 92.6 million patients with DR and 20.6 million DME patients, with the number of sufferers expected to rise in the future (Yau et al. 2012). The relationship between DR and Hemoglobin A1c (HbA1c) was revealed in the landmark Diabetes Control and Complications Trial (DCCT) study (The Diabetes Control and Complications Trial Research Group 1996). Therefore, sustained lowering of blood glucose levels can prevent the risk of progression of DR and other diabetic complications. As such, numerous anti-diabetic drugs have been developed by pharmaceutical companies and seven different types of agents are now available: sulfonylureas, biguanides, $\alpha$-glucosidase inhibitors, glinides, thiazolidinediones, incretin related drugs and sodium-glucose cotransporter (SGLT) 2 inhibitors (Takamoto and Kadowaki 2011, Chen et al. 2016). However, despite the numerous available choices, diabetic complications often develop and lead to both 
life-threatening and life-altering problems.

Many years of diabetes leads to biochemical and molecular abnormalities, such as the production of advanced glycation end-products (AGEs), generation of reactive oxygen species (ROS) and activation of protein kinase $\mathrm{C}$ (PKC) followed by cytokines, including vascular endothelial growth factor (VEGF), and chemokine production in retina and retinal capillaries (Das 2016).

VEGF, as a permeable factor, induces the alteration of tight junction proteins that contributes to a breakdown of the blood-retinal barrier (BRB), promotes vascular permeability and causes retinal edema (Kim et al. 2009, Murakami et al. 2009). Hence, neutralizing anti-VEGF antibodies, such as ranibizumab, bevacizumab and VEGF trap, have become standard of care for DME in the past 10 years. However, $25 \%$ of DME patients do not respond to anti-VEGF treatment (Singer et al. 2016). Therefore, the elucidation of the underlying mechanism is highly anticipated. To reveal the mechanism, a few animal models have been investigated to date. Streptozotocin (STZ) induced rats (Yu et al. 2010) or mice (Anand-Apte et al. 2010), Otsuka Long-Evans Tokushima Fatty (OLETF) rats (Lu et al. 2003), and Zucker Diabetic Fatty (ZDF) rats (Johnson et al. 2013) are often used. However, retinal thickening is not observed in these animals, unlike human DME patients.

The Spontaneously Diabetic Torii (SDT) fatty rat was established by introducing the fa allele into the SDT rat genome, and the onset of diabetes is observed at 6 weeks of age (Masuyama et al. 2005). Recent studies indicate that this model also exhibits various diabetic complications (Katsuda et al. 2015). In particular, in the retina, the peak latencies of oscillatory potentials in electroretinograms (ERGs) at 16 weeks of age are prolonged when compared with the lean rat, demonstrating retinal dysfunction (Matsui et al. 2008). In this study, we investigated pathophysiological changes associated with hyperglycemia in the retina of SDT fatty rats. We evaluated VEGF accumulation in the vitreous humor, retinal vascular permeability, retinal thickening (Experiment 1), and the therapeutic effects of anti-diabetic drugs on retinal thickening in the male SDT fatty rat (Experiment 2).

\section{Methods}

Animals

Male SDT $f a / f a$ (fatty) rats and age-matched Sprague-Dawley (SD) rats were purchased from CLEA
Japan, Inc. (Tokyo, Japan). Rats were housed in a climate-controlled room at a temperature of $23 \pm 3{ }^{\circ} \mathrm{C}$, humidity of $55 \pm 15 \%$, and a $12 \mathrm{~h}$ lighting cycle. A powdered basal diet (CRF-1, Oriental Yeast Co., Ltd., Tokyo, Japan) and water were provided ad libitum. All experiments received prior approval from the committee for human care and use of animals at our laboratory in accordance with the Standards Relating to the Care and Management of Experimental Animals.

\section{Biological parameters}

Body weight and biological parameters were evaluated. Blood samples were collected from the tail vein. Glucose, HbAlc, triglyceride and total cholesterol levels were measured using commercial kits (Roche Diagnostics, Basel, Switzerland) and an automatic analyzer (Hitachi 7180; Hitachi, Tokyo, Japan). Plasma insulin levels were measured using an ELISA kit (Morinaga, Yokohama, Japan).

\section{Experiment 1}

VEGF concentration in vitreous humor

Animals at 8 and 16 weeks of age were sacrificed under deep isoflurane anesthesia. Both eyes were enucleated, washed with saline three times, and then wiped with cotton to dry. After pricking the eyes near the optic nerve with a 27-gauge needle, approximately $60 \mu \mathrm{l}$ of the vitreous humor was collected into $0.5 \mathrm{ml}$ Eppendorf tubes and immediately frozen in liquid nitrogen. Samples were thawed on ice and centrifuged at $10,000 \mathrm{xg}$ for $5 \mathrm{~min}$ at $4{ }^{\circ} \mathrm{C}$, after which the supernatant was used. VEGF concentrations were measured using a Rat VEGF Quantikine ELISA Kit (R\&D Systems Inc., Minneapolis, MN, USA).

\section{Retinal vascular permeability}

Rats were given an intravenous dose of $10 \mathrm{mg} / \mathrm{kg}$ of sodium fluorescein. One hour later, blood samples and vitreous humor were collected. After centrifugation $\left(10,000 \mathrm{xg}\right.$ for $5 \mathrm{~min}$ at $\left.4{ }^{\circ} \mathrm{C}\right)$, the fluorescein concentration of the serum samples was measured by using a microplate reader (ARVO-X5 2030. Multilabel Reader; Perkin-Elmer) with excitation at $485 \mathrm{~nm}$ and emission at $535 \mathrm{~nm}$. Retinal vascular permeability was calculated by dividing fluorescein concentration of vitreous humor by that of blood plasma.

\section{Ocular histopathology}

The eyes were enucleated and fixed in $1 \%$ 
formalin $/ 1.5 \%$ glutaraldehyde mix fixative solution, and then embedded in paraffin, sectioned, stained with hematoxylin and eosin (HE), and examined histopathologically.

\section{Measurement of retinal thickness}

In the previous report by Toyoda et al. (2016), mean retinal thickness at 500,1,000, and 1,500 $\mu \mathrm{m}$ from the optic nerve disc were almost same. Therefore, the total retinal thickness in this experiment was defined as the distance between the retinal internal limiting membrane (ILM) and the photoreceptor layer (PL) $500 \mu \mathrm{m}$ away from the optic nerve disc. The thickness of the ILM of the ganglion cell layer (GCL), the inner plexiform layer (IPL), inner nuclear layer (INL), outer nuclear layer (ONL) and PL were also measured.

\section{Experiment 2}

Experiment grouping design for anti-diabetic drugs

Based on the biological parameters, SD rats and SDT fatty rats at 4 weeks of age were divided into four subgroups; Group 1 (normal control): SD rats subcutaneously injected with $20 \%$ propylene glycol and fed powdered CRF-1 chow; Group 2 (DME vehicle): SDT fatty rats subcutaneously injected with $20 \%$ propylene glycol and fed powdered CRF-1 chow; Group 3 (DME pioglitazone): SDT fatty rats subcutaneously injected with $20 \%$ propylene glycol and administered $10 \mathrm{mg} / \mathrm{kg} /$ day pioglitazone (synthesized at
Japan Tobacco Co.) through powdered CRF-1 chow (food admixture); and Group 4 (DME phlorizin): SDT fatty rats subcutaneously injected with $150 \mathrm{mg} / \mathrm{kg} /$ day phlorizin (Sigma-Aldrich Co., St. Louis, MO, USA) and fed powdered CRF-1 chow.

\section{Statistical analysis}

All values were expressed as the mean \pm standard deviation. If there were 2 groups to compare, an F-test for homogeneity of variance followed by Student's t-test or Aspin-Welch's t-test was performed. To compare the means of the three groups (Experiment 2), Dunnett's test or Steel's test was performed after Bartlett's test was performed to assess the homogeneity of variances. All statistical analyses were performed using Statlight 2000 (Yukms Corp., Tokyo, Japan) statistical software. A $p<0.05$ was considered statistically significant.

\section{Results}

\section{Experiment 1}

Body weight and blood chemical parameters, such as glucose, insulin, triglyceride (TG) and total cholesterol (TC) levels, are shown in Table 1. At 8 weeks of age, the SDT fatty rats exhibited obesity, hyperglycemia, hyperinsulinemia and hyperlipidemia. Although hyperglycemia was observed in SDT fatty rats that were 16 weeks of age, body weight, insulin and TG levels showed decreases.

Table 1. Body weight and serum biochemical parameters.

\begin{tabular}{lcccc}
\hline & \multicolumn{2}{c}{ SD rat } & \multicolumn{2}{c}{ SDT fatty rat } \\
Parameters & 8 weeks & $\mathbf{1 6}$ weeks & 8 weeks & 16 weeks \\
& & & & \\
Body weight $(g)$ & $342.5 \pm 8.6$ & $625.8 \pm 40.3^{\# \#}$ & $390.6 \pm 27.4^{*}$ & $510.4 \pm 36.7^{* * \# \#}$ \\
Glucose $(\mathrm{mg} / \mathrm{dl})$ & $148.8 \pm 10.5$ & $196.2 \pm 14.0^{\# \#}$ & $520.8 \pm 162.4^{* *}$ & $741.6 \pm 76.9^{* * \#}$ \\
Insulin $(\mathrm{ng} / \mathrm{ml})$ & $0.73 \pm 0.17$ & $0.84 \pm 0.17$ & $8.3 \pm 5.4^{*}$ & $1.1 \pm 0.2^{\#}$ \\
TG $(\mathrm{mg} / \mathrm{dl})$ & $154.6 \pm 58.1$ & $215.3 \pm 72.8$ & $764.0 \pm 150.8^{* *}$ & $330.5 \pm 86.7^{\# \#}$ \\
TC $(\mathrm{mg} / \mathrm{dl})$ & $77.2 \pm 8.9$ & $64.7 \pm 4.8^{\#}$ & $106.9 \pm 7.1^{* *}$ & $136.5 \pm 27.7^{* *}$ \\
\hline
\end{tabular}

Body weight and plasma glucose, insulin, triglyceride (TG) and total cholesterol (TC) levels in SD rats and SDT fatty rats were measured at 8 and 16 weeks of age. SDT fatty rats exhibited significant increases in body weight, plasma glucose, insulin, TG and TC at 8 weeks of age. At 16 weeks of age, SDT fatty rats exhibited severe hyperglycemia with decreases in plasma insulin and TG levels. Data are presented as the mean \pm S.D. $(n=5) . * p<0.05, * * p<0.01$ versus age-matched SD rats (t-test). ${ }^{*} p<0.05,{ }^{\# \#} p<0.01$, significantly different from rats 8 weeks of age.

The vitreous humor from SD rats and SDT fatty rats were collected. Compared with normal values, the mean vitreous VEGF concentration value in SDT fatty rats was significantly higher at 8 weeks of age $(50.5 \pm 19.2 \mathrm{pg} / \mathrm{ml}$ for SD rats versus $200.1 \pm 41.9 \mathrm{pg} / \mathrm{ml}$ for SDT fatty rats) and showed tendency to increase at 
16 weeks of age $(9.0 \pm 2.7 \mathrm{pg} / \mathrm{ml}$ for $\mathrm{SD}$ rats versus $28.6 \pm 32.6 \mathrm{pg} / \mathrm{ml}$ for SDT fatty rats) in Figure 1A. Furthermore, the retinal permeability of SDT fatty rats at
16 weeks of age also showed tendency to increase ( $p$ value $=0.07)$ in Figure 1B.
A

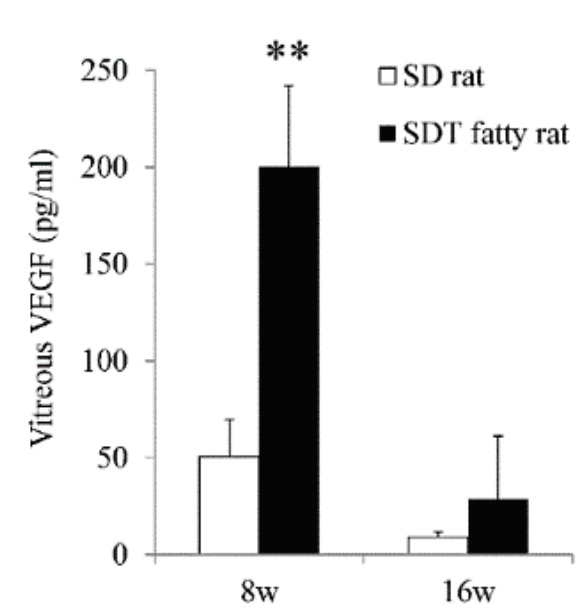

B

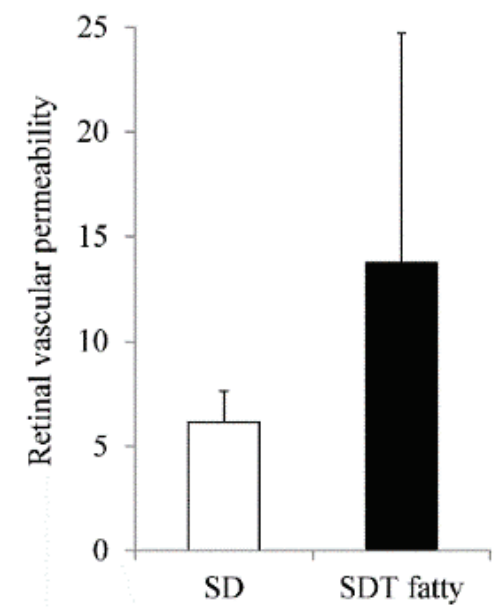

Fig. 1. VEGF concentrations in the vitreous humor (A) and retinal vascular permeability (B) of SD rats and SDT fatty rats at 8 and 16 weeks of age were collected and measured. Each value represents the mean \pm S.D. $(A: n=4, B: n=8)$. $* * p<0.01$, significantly different from SD rats.
The eye specimens from SD rats (Fig. 2A) and SDT fatty rats (Fig. 2B) were observed under a microscope. The mean values for total retinal thickness, defined as the distance from the ILM to the PL $500 \mu \mathrm{m}$ away from the optic nerve disc, were measured. The retinas of SDT fatty rats were significantly thicker than that of SD rats in Figure 2C $(157.5 \pm 19.8 \mu \mathrm{m}$ for SD rats versus $218.4 \pm 16.9 \mu \mathrm{m}$ for $\mathrm{SD}$ rats). The layers between the ILM and the GCL, the IPL, INL and ONL of SDT fatty rats were significantly thicker than those of SD rats. On the other hand, the thickness of the PL in SDT fatty rats was not significant when compared with that in SD rats, as shown in Figure 2D.

\section{Experiment 2}

SDT fatty rats were treated from 4 to 16 weeks of age with anti-diabetic drugs, pioglitazone (10 mg/kg/day) or phlorizin (150 mg/kg/day). Pioglitazone significantly increased body weight at 8 and 16 weeks of age and phlorizin at 16 weeks of age (Fig. 3A). Both drugs strongly reduced plasma glucose levels (Fig. 3B), HbAlc (Fig. 3C) and TG levels (Fig. 3E) at 8 and 16 weeks of age. From 8 to 16 weeks of age, vehicle groups exhibited sharp declines in plasma insulin levels, whereas both treatment groups retained higher levels (Fig. 3D), indicating the preservation of pancreatic $\beta$-cell function. For plasma TC levels, treatment with pioglitazone resulted in a tendency toward decreases and significant decreases at 16 weeks of age (Fig. 3F) with phlorizin treatment.

The total retina of SDT fatty rats was thicker than that of SD rats, and both pioglitazone and phlorizin decreased retinal thickness in Figure 4A. The layers between the ILM and GCL, the IPL, INL and ONL in SDT fatty rats were significantly thicker than those of SD rats and these layers showed a tendency to decrease when treated using either of the drugs in Figure 4B.

\section{Discussion}

To reveal the mechanism and develop new therapies, many researchers have investigated animal models. STZ-induced animals are recognized as the most common model and retinal hyperpermeability was reported. Nevertheless, in this model, retinal thickness becomes thinner than control, unlike in human patients (Zhang et al. 2008). Although the OLETF rat and ZDF rat show morphological changes in the retinal capillaries or pericytes, retinal thickening has unfortunately not been reported (Lu et al. 2003, Johnson et al. 2013, Lai and Lo 2013).

The SDT rat is a known non-obese type 2 diabetes model and VEGF overexpression, vascular lesions, such as acellular capillaries and pericyte loss, leakage of fluorescein around the optic disc, retinal thickening, neovascularization, and tractional retinal detachment with fibrous proliferation around 60 weeks of ages have been reported by Kakehashi et al. (Shinohara et 
A

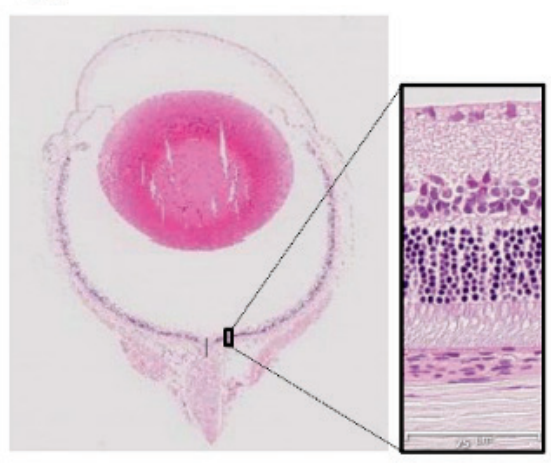

B

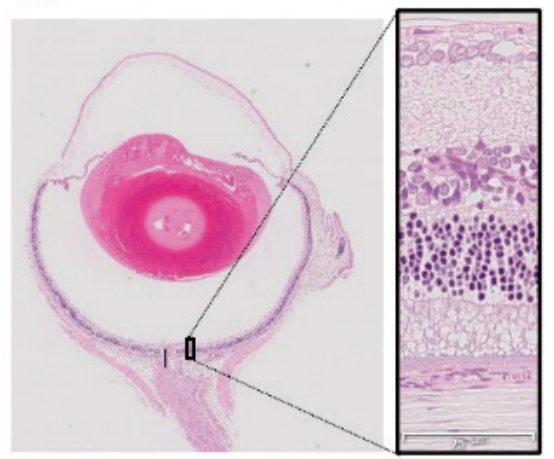

$\mathrm{C}$

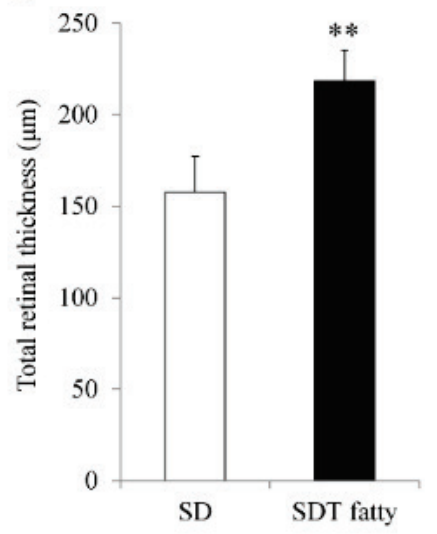

D

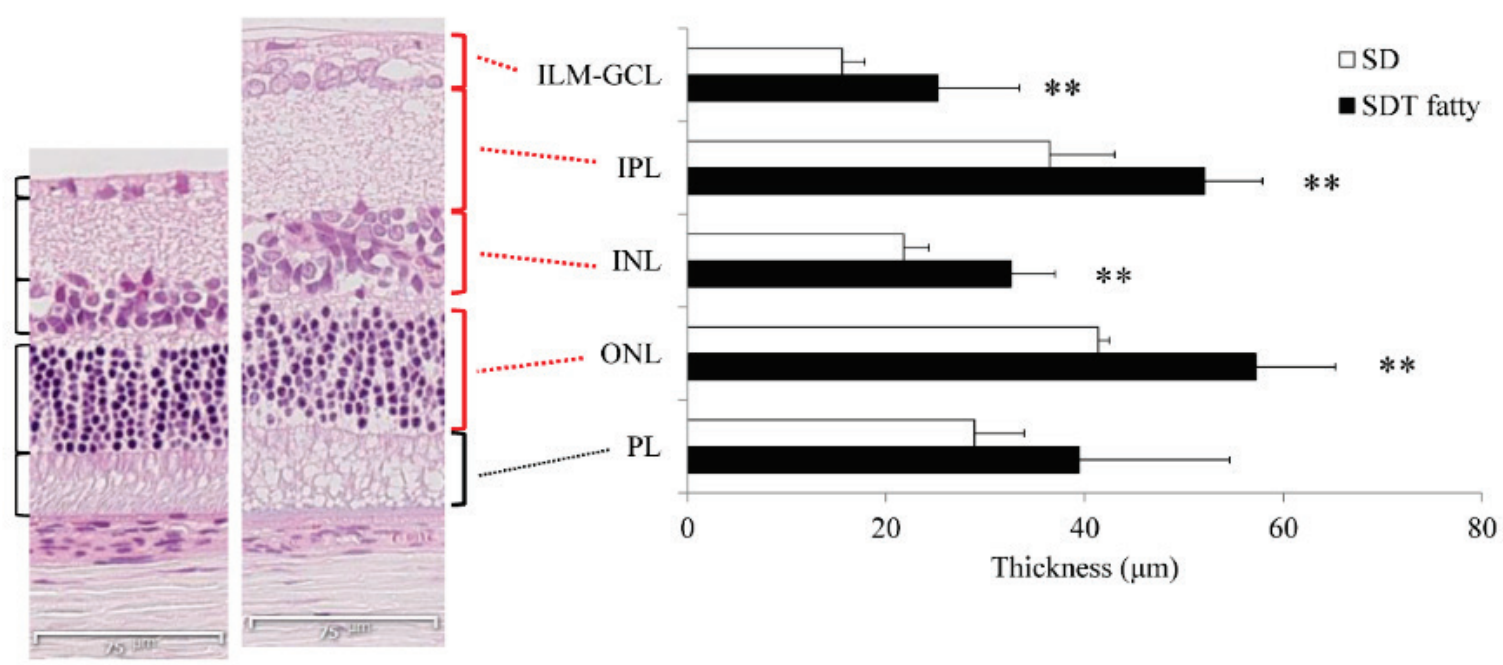

Fig. 2. Photomicrograph of the eyes of male SD rats (A, representative example) and SDT fatty rats (B, representative example) at 16 weeks of age. The layer in the retina that was $500 \mu \mathrm{m}$ from the optic disc was enlarged. The total retinal thickness (C) and the layer between the ILM (inter limiting membrane) and GCL (ganglion cell layer), the IPL (inner plexiform layer), INL (inner nuclear layer), ONL (outer nuclear layer) and PL (photoreceptor layer) (D) were evaluated. Each value represents the mean \pm S.D. $(n=5)$. ${ }^{* *} p<0.01$, significantly different from SD rats.

al. 2000, Kakehashi et al. 2006, Sasase et al. 2013, Toyoda et al. 2016). However, these retinal abnormalities develop over the course of a year. Because SDT fatty rat was originally produced by introducing $f a$ gene to SDT rat to accelerate the development of diabetic complications, similar ocular lesions are basically expected between these two strains. However, the effect of aging is quite important for pathophysiology of eyes actually, SD rats demonstrate spontaneous ocular abnormalities, such as corneal or lenticular opacities, choroidal or retinal atrophy, retinal fold, and hemorrhage increased with greater frequency with increasing age (Ban et al. 2008, Tomomi 2008). Therefore, advancing the development of ocular complications is meaningful for studying diabetic retinopathy using experimental animal model. In addition, according to obesity and following insulin resistance, inflammation is found in eyes in SDT fatty rat at older age (Kemmochi et al. 2014). Also, further studies are needed to unveil the difference of response to various drugs, such as SGLT2 inhibitor, between SDT and SDT fatty rat.

The SDT fatty rat is recognized as a diabetes model, and in this study, exhibited severe hyperglycemia from 8 weeks of age followed by VEGF overproduction, retinal vascular hyperpermeability, and retinal thickening. Generally, rapid increases in blood glucose may enhance glycolysis and the diacylglycerol pathway involving PKC activation followed by VEGF production (Xia et al. 2007). Furthermore, oxidative stress induced by hyperglycemia also causes VEGF production, matrix metalloproteinase (MMP) activation and breakdowns in the BRB (El-Remessy et al. 2013). These changes can be 
A

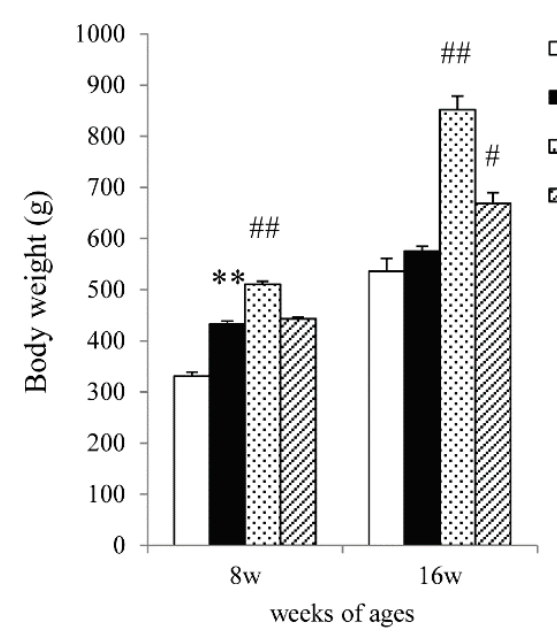

C

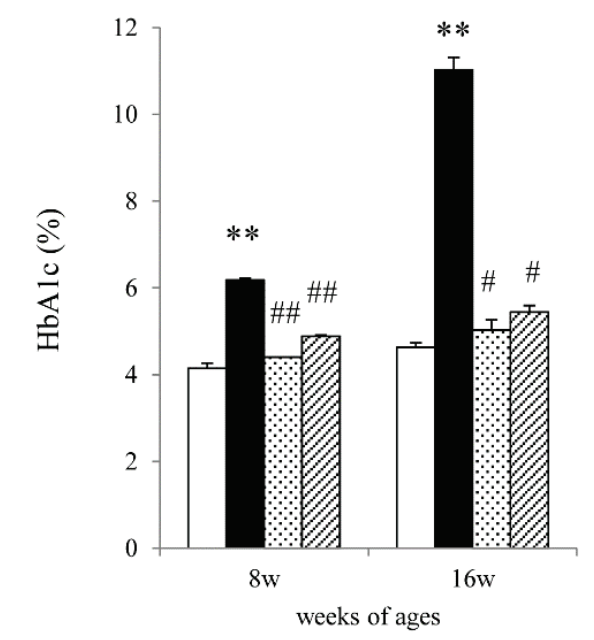

E

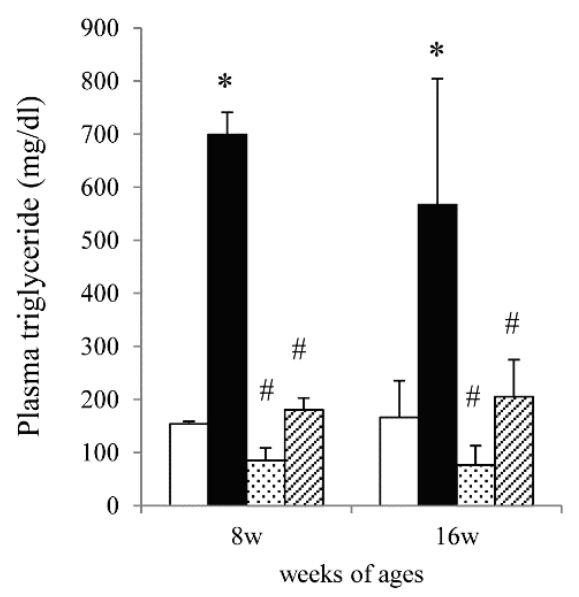

B

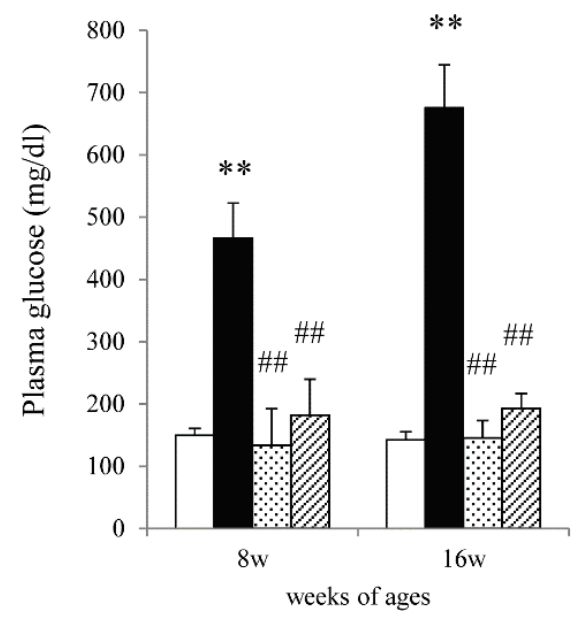

D

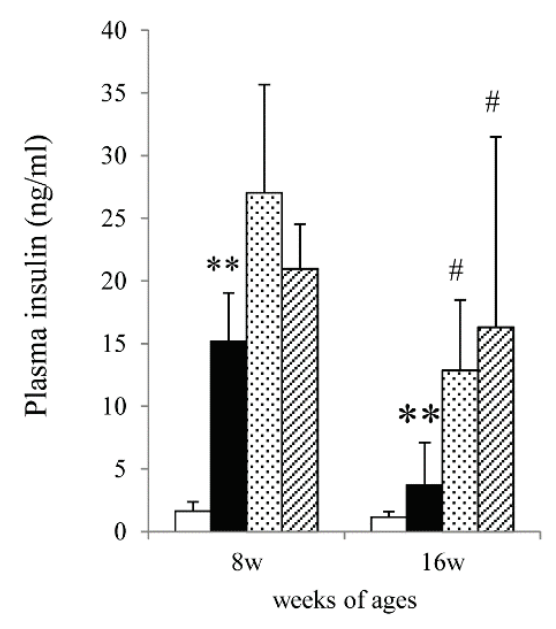

F

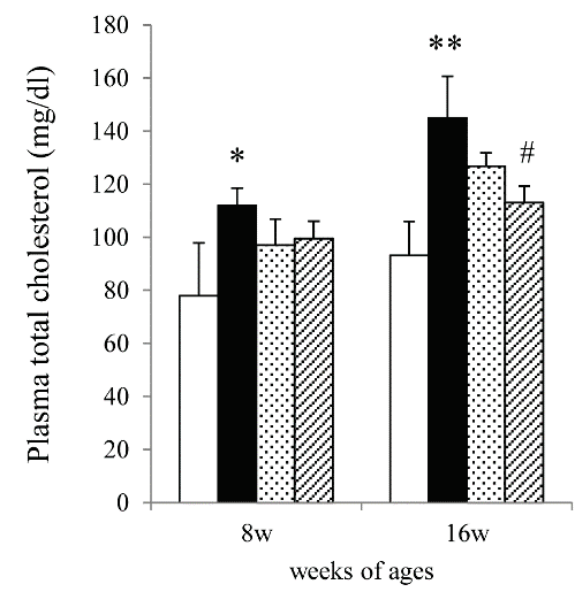

Fig. 3. Effect of pioglitazone and phlorizin on body weight and blood biological parameters. SDT fatty rats were treated with pioglitazone $(10 \mathrm{mg} / \mathrm{kg} /$ day $)$ or phlorizin $(150 \mathrm{mg} / \mathrm{kg} /$ day) from 4 to 16 weeks of age. Body weight (A) and plasma glucose level (B), HbA1c level (C), plasma insulin (D), triglyceride (TG) (E) and total cholesterol (TC) levels (F) at 8 and 16 weeks of ages were evaluated. Each value represents the mean \pm S.D. $(\mathrm{n}=4)$. ${ }^{*} p<0.05, * * p<0.01$, significantly different from SD rats. ${ }^{\#} p<0.05$, \#\# $p<0.01$, significantly different from the vehicle group. 
A

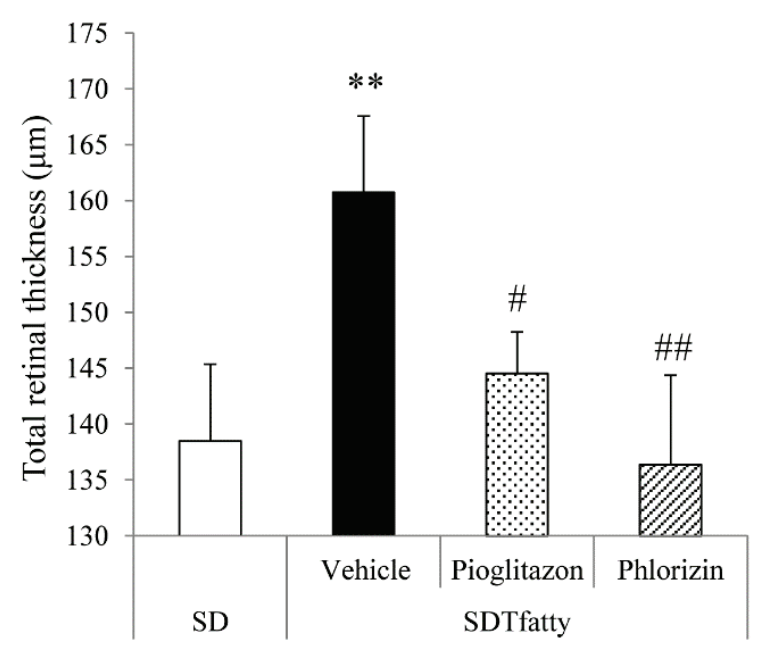

B

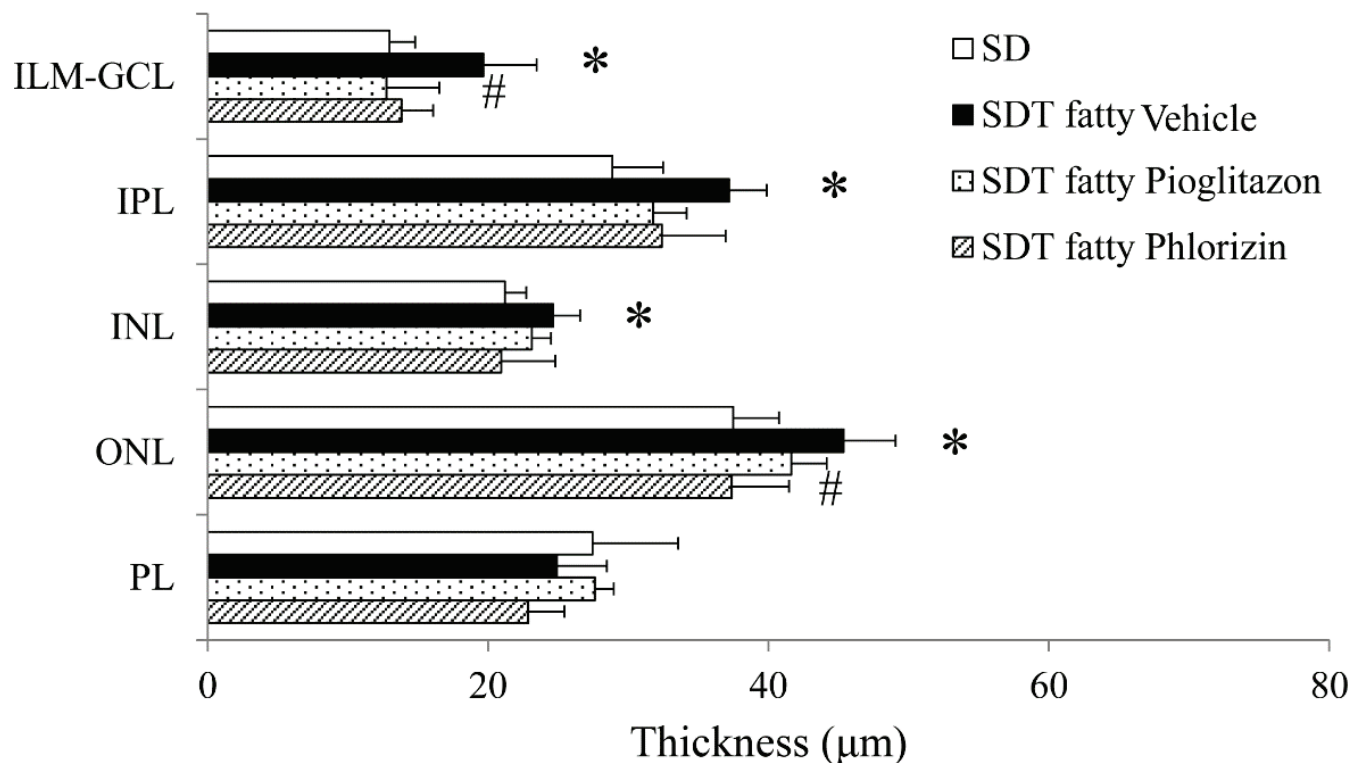

Fig. 4. Effect of pioglitazone and phlorizin on retinal thickening. The total retinal thickness (A) and layers, such as between the ILM and $\mathrm{GCL}$, the IPL, INL, ONL and PL (B) that is $500 \mu \mathrm{m}$ from the optic disc, were measured. Pioglitazone and phlorizin improved total retinal thickness, in particular, from the ILM to the ONL. Each value represents the mean \pm S.D. $(n=4) . * * p<0.01$, significantly different from the SD rats. ${ }^{\#} p<0.05,{ }^{\# \#} p<0.01$, significantly different from the vehicle group.

one of the reasons of the peak latency in ERGs of male SDT fatty rats at 16 weeks of age (Matsui et al. 2008).

In this study, we have chosen pioglitazone and phlorizin to evaluate the effect of controlling blood glucose level. These drugs are cheap and easy to purchase, and above all, these drugs have already reported to lower blood glucose levels well without any side effects in SDT fatty rats. Interestingly, although the glucose-lowering effect of pioglitazone and phlorizin is the same, pioglitazone could not normalize retinal thickness. Recently, Dennis et al. (2015) reported that reductions in serum glucose concentrations with phlorizin treatment led to normalized VEGF concentrations in the retina of STZ-induced rats. Therefore, glucose-lowering effects can contribute to decreases in retinal VEGF expression. 
Generally, glitazones have side effects, such as edema, which is likely caused by renal sodium retention (Colucciello 2005, Ryan et al. 2006, Horita et al. 2015), and in actual human DME patients, some studies have indicated the adverse effect of pioglitazone (Oshitari et al. 2008, Idris et al. 2012).

Hence, the effect of pioglitazone must be weakened against the retinal thickness despite plasma glucose normalization. As mentioned above, lowering blood glucose levels normalize VEGF concentrations in retina. On the other hands, pioglitazone causes edema as its side effect. Therefore, we speculate that the imbalance of effect and side effect of pioglitazone may cause different output on retinal thickness between human and SDT fatty rat. Given these facts, SDT fatty rat is a prominent animal model, which enables us to evaluate not only positive effects but also side effects in advance without clinical trials.

In summary, we identified an increase in VEGF in vitreous humor, retinal vascular hyperpermeability, and retinal thickening, in particular from the ILM to ONL of SDT fatty rats at 16 weeks of age. Furthermore, the retinal thickening improved with treatment with anti-diabetic drugs, pioglitazone and phlorizin. However, unlike phlorizin, the effect of pioglitazone is not strong despite the normalization of blood glucose levels. Altogether, we confirmed that the male SDT fatty rat is a suitable preclinical model for testing new therapeutic approaches in DME.

\section{Conflict of Interest}

There is no conflict of interest.

\section{References}

ANAND-APTE B, EBRAHEM Q, CUTLER A, FARAGE E, SUGIMOTO M, HOLLYFIELD J, FOLKMAN J: Betacellulin induces increased retinal vascular permeability in mice. PLoS One 5: e13444, 2010.

BAN Y, TOMOHIRO M, INAGAKI S, KUNO H: Spontaneous ocular abnormalities in Crl:CD(SD) rats. Anim Eye Res 27: 9-15, 2008.

CHEN M, XIE CG, GAO H, ZHENG H, CHEN Q, FANG JQ: Comparative effectiveness of sodium-glucose cotransporter 2 inhibitors for controlling hyperglycaemia in patients with type 2 diabetes: protocol for a systematic review and network meta-analysis. BMJ Open 6: e010252, 2016.

CHEUNG N, MITCHELL P, WONG TY: Diabetic retinopathy. Lancet 376: 124-136, 2010.

COLUCCIELLO M: Vision loss due to macular edema induced by rosiglitazone treatment of diabetes mellitus. Arch Ophthalmol 123: 1273-1275, 2005.

DAS A: Diabetic retinopathy: battling the global epidemic. Invest Ophthalmol Vis Sci 57: 6669-6682, 2016.

DENNIS MD, KIMBALL SR, FORT PE, JEFFERSON LS: Regulated in development and DNA damage 1 is necessary for hyperglycemia-induced vascular endothelial growth factor expression in the retina of diabetic rodents. J Biol Chem 290: 3865-3874, 2015.

EL-REMESSY AB, FRANKLIN T, GHALEY N, YANG J, BRANDS MW, CALDWELL RB, BEHZADIAN MA: Diabetes-induced superoxide anion and breakdown of the blood-retinal barrier: role of the VEGF/uPAR pathway. PLoS One 8: e71868, 2013.

HORITA S, NAKAMURA M, SATOH N, SUZUKI M, SEKI G: Thiazolidinediones and edema: recent advances in the pathogenesis of thiazolidinediones-induced renal sodium retention. PPAR Res 2015: 646423, 2015.

IDRIS I, WARREN G, DONNELLY R: Association between thiazolidinedione treatment and risk of macular edema among patients with type 2 diabetes. Arch Intern Med 172: 1005-1011, 2012.

JOHNSON LE, LARSEN M, PEREZ MT: Retinal adaptation to changing glycemic levels in a rat model of type 2 diabetes. PLoS One 8: e55456, 2013.

KAKEHASHI A, SAITO Y, MORI K, SUGI N, ONO R, YAMAGAMI H, SHINOHARA M, TAMEMOTO H, ISHIKAWA SE, KAWAKAMI M, KANAZAWA Y: Characteristics of diabetic retinopathy in SDT rats. Diabetes Metab Res Rev 22: 455-461, 2006.

KATSUDA Y, SASASE T, TADAKI H, MERA Y, MOTOHASHI Y, KEMMOCHI Y, TOYODA K, KAKIMOTO K, KUME S, OHTA T: Contribution of hyperglycemia on diabetic complications in obese type 2 diabetic SDT fatty rats: effects of SGLT inhibitor phlorizin. Exp Anim 64: 161-169, 2015. 
KEMMOCHI Y, MIYAJIMA K, OHTA T, SASASE T, YASUI Y, TOYODA K, KAKIMOTO K, SHODA T, KAKEHASHI A: Ocular inflammation in uveal tract in aged obese type 2 diabetic rats (spontaneously diabetic Torii fatty rats). J Diabetes Res 2014: 629016, 2014.

KIM JH, KIM JH, LEE YM, AHN EM, KIM K, WYU YS: Decursin inhibits VEGF-mediated inner blood-retinal barrier breakdown by suppression of VEGFR-2 activation. J Cereb Blood Flow Metab 29: 1559-1567, 2009.

LAI AK, LO AC: Animal models of diabetic retinopathy: summary and comparison. J Diabetes Res 2013: 106594, 2013.

LU ZY, BHUTTO IA, AMEMIYA T: Retinal changes in Otsuka long-evans Tokushima Fatty rats (spontaneously diabetic rat)--possibility of a new experimental model for diabetic retinopathy. Jpn J Ophthalmol 47: 28-35, 2003.

MASUYAMA T, KATSUDA Y, SHINOHARA M: A novel model of obesity-related diabetes: introgression of the Lepr(fa) allele of the Zucker fatty rat into nonobese Spontaneously Diabetic Torii (SDT) rats. Exp Anim 54: 13-20, 2005.

MATSUI K, OHTA T, ODA T, SASASE T, UEDA N, MIYAJIMA K, MASUYAMA T, SHINOHARA M, MATSUSHITA M: Diabetes-associated complications in Spontaneously Diabetic Torii fatty rats. Exp Anim 57: 111-121, 2008.

MURAKAMI T, FELINSKI EA, ANTONETTI DA: Occludin phosphorylation and ubiquitination regulate tight junction trafficking and vascular endothelial growth factor-induced permeability. $J$ Biol Chem 284: 21036-21046, 2009.

OSHITARI T, ASAUMI N, WATANABE M, KUMAGAI K, MITAMURA Y: Severe macular edema induced by pioglitazone in a patient with diabetic retinopathy: a case study. Vasc Health Risk Manag 4: 1137-1140, 2008.

RYAN EH JR, HAN DP, RAMSAY RC, CANTRILL HL, BENNETT SR, DEV S, WILLIAMS DF: Diabetic macular edema associated with glitazone use. Retina 26: 562-570, 2006.

SASASE T, OHTA T, MASUYAMA T, YOKOI N, KAKEHASHI A, SHINOHARA M: The spontaneously diabetic torii rat: an animal model of nonobese type 2 diabetes with severe diabetic complications. J Diabetes Res 2013: 976209, 2013.

SHINOHARA M, MASUYAMA T, SHODA T, TAKAHASHI T, KATSUDA Y, KOMEDA K, KUROKI M, KAKEHASHI A, KANAZAWA Y: A new spontaneously diabetic non-obese Torii rat strain with severe ocular complications. Int J Exp Diabetes Res 1: 89-100, 2000.

SINGER MA, KERMANY DS, WATERS J, JANSEN ME, TYLER L: Diabetic macular edema: it is more than just VEGF. F1000Res 5, 2016. doi: 10.12688/f1000research.8265.1.

TAKAMOTO I, KADOWAKI T: Treatment of diabetes mellitus with oral hypoglycemic agents. (In Japanese) Nihon Rinsho 69: 563-572, 2011.

THE DIABETES CONTROL AND COMPLICATIONS TRIAL RESEARCH GROUP: The absence of a glycemic threshold for the development of long-term complications: the perspective of the diabetes control and complications trial. Diabetes 45: 1289-1298, 1996.

TOMOMI M: Spontaneous hemorrhagic findings in fundus of Sprague-Dawley and Fischer rats. Anim Eye Res 27: 31-37, 2008.

TOYODA F, TANAKA Y, SHIMMURA M, KINOSHITA N, TAKANO H, KAKEHASHI A: Diabetic retinal and choroidal edema in SDT Rats. J Diabetes Res 2016: 2345141, 2016.

XIA L, WANG H, MUNK S, FRECKER H, GOLDBERG HJ, FANTUS IG, WHITESIDE CI: Reactive oxygen species, PKC-beta1, and PKC-zeta mediate high-glucose-induced vascular endothelial growth factor expression in mesangial cells. Am J Physiol Endocrinol Metab 293: E1280-E1288, 2007.

YAU JW, ROGERS SL, KAWASAKI R, LAMOUREUX EL, KOWALSKI JW, BEK T, CHEN SJ, DEKKER JM, FLETCHER A, GRAUSLUND J, HAFFNER S, HAMMAN RF, IKRAM MK, KAYAMA T, KLEIN BE, ET AL.: Global prevalence and major risk factors of diabetic retinopathy. Diabetes Care 35: 556-564, 2012.

YU H, CHEN L, JIANG J: Administration of pigment epithelium-derived factor delivered by adeno-associated virus inhibits blood-retinal barrier breakdown in diabetic rats. Mol Vis 16: 2384-2394, 2010. 
ZHANG J, WU Y, JIN Y, JI F, SINCLAIR SH, LUO Y, XU G, LU L, DAI W, YANOFF M, LI W, XU GT: Intravitreal injection of erythropoietin protects both retinal vascular and neuronal cells in early diabetes. Invest Ophthalmol Vis Sci 49: 732-742, 2008. 